\title{
Epidemiology of Staphylococcus aureus Infections in Kenya: Current State, Gaps and Opportunities
}

\author{
Justin Nyasinga ${ }^{1,2,3}$, Geoffrey Omuse ${ }^{1}$, Njenga John' ${ }^{1}$, Andrew Nyerere ${ }^{3}$, Shima Abdulgader ${ }^{4}$, \\ Mae Newton ${ }^{4}$, Andrew Whitelaw ${ }^{4}$, Gunturu Revathi ${ }^{*}$ \\ ${ }^{1}$ Department of Pathology, Aga Khan University, Nairobi, Kenya \\ ${ }^{2}$ Department of Biomedical Sciences and Technology, The Technical University of Kenya, Nairobi, Kenya \\ ${ }^{3}$ Pan-African University-Institute of Science, Technology and Innovation/Jomo Kenyatta University of Agriculture and \\ Technology, Nairobi, Kenya \\ ${ }^{4}$ Department of Biomedical Sciences, Stellenbosch University, Stellenbosch, South Africa \\ Email: *gunturu.revathi@aku.edu
}

How to cite this paper: Nyasinga, J., Omuse, G., John, N., Nyerere, A., Abdulgader, S., Newton, M., Whitelaw, A. and Revathi, G. (2020) Epidemiology of Staphylococcus aureus Infections in Kenya: Current State, Gaps and Opportunities. Open Journal of Medical Microbiology, 10, 204-221.

https://doi.org/10.4236/ojmm.2020.104018

Received: October 29, 2020

Accepted: December 15, 2020

Published: December 18, 2020

Copyright (c) 2020 by author(s) and Scientific Research Publishing Inc. This work is licensed under the Creative Commons Attribution International License (CC BY 4.0).

http://creativecommons.org/licenses/by/4.0/

\begin{abstract}
Staphylococcus aureus has maintained its clinical relevance as a major cause of hospital and community acquired infections globally with a high burden of antimicrobial resistance (AMR). Though reported, the burden of infection, antimicrobial resistance and molecular epidemiology of $S$. aureus are not well defined in Kenya. This descriptive review evaluated reported data on the detection and characterization of $S$. aureus infections in Kenya. Published data between 2000 and 2020 were evaluated. $S$. aureus isolation frequencies varied from $1 \%$ in blood specimens to $52.6 \%$ among skin and soft tissues infections while MRSA rates ranged from $1 \%$ to $84.1 \%$. While penicillin resistance has consistently been high, last line and recent antibiotics such as vancomycin, linezolid, teicoplanin and daptomycin have retained their efficacy. Data on MRSA carriage in the community, among HCWs and inpatients is limited. Global clones (CC1, CC5, CC8, CC22, CC30, CC45 and CC239) alongside a few novel MRSA strains have been reported with staphylococcal protein A (spa) sequence based clustering yielding four major clusters ( $s p a$ CC359, spa CC005, spa CC121 and spa CC021) in circulation. MRSA strain ST239/241 (t037) seems predominant in the country. Despite a clear paucity of data, the present analysis points to a high infection and AMR burden in $S$. aureus with global MRSA clones in circulation. Standardized national surveillance and reporting incorporating molecular tools for identification and characterization will help fill existing gaps in the understanding of the evolving epidemiology of MRSA infections.
\end{abstract}




\section{Keywords}

Staphylococcus aureus, MRSA, AMR, Epidemiology, Kenya

\section{Introduction}

Staphylococcus aureus is one of the most frequently encountered pathogens in clinical settings as a cause of a wide range of infections of varying severity which include boils, cellulitis, food poisoning, sepsis, osteomyelitis, pneumonia and endocarditis [1]. Evolution and spread of antimicrobial resistance present a major challenge in the control of infections associated with the pathogen. Methicillin resistant $S$. aureus (MRSA) strains are especially troublesome as they may be associated with increased morbidity, a heightened risk of mortality, longer hospital stays and higher healthcare costs [2].

The first WHO report on global AMR surveillance identified MRSA prevalence of at least $50 \%$ in five of the six WHO regions [2]. Some MRSA strains have emerged as sources of infections in apparently healthy individuals in the community, a clear departure from their traditional association with healthcare settings among high risk patient populations [3]. The ever growing resistance coupled with a declining pipeline of new antimicrobials and a lack of an effective anti-staphylococcal vaccine necessitate concerted efforts in AMR surveillance, drug and vaccine discovery [4].

Staphylococcal infections and associated antimicrobial resistance have been reported in different settings in Africa in spite of disparities within and between countries [5] [6]. However, the WHO report on global AMR surveillance identified a lack of adequate data from the WHO Africa region as a major challenge in the estimation of the burden of AMR. A recent analysis of the clinical and economic burden of AMR in developing countries found a lack of sufficient data from Africa to warrant any analyses [7]. Moreover, there is limited data on the molecular epidemiology of drug-resistant $S$. aureus infections in Africa with inadequate technical capacity, high costs and complexity of existing molecular tools cited as challenges [8] [9].

To contribute towards filling the current gaps in the epidemiology of $S$. aureus infections in Kenya, we have reviewed 20-year published data with respect to $S$. aureus isolation frequencies, antimicrobial resistance profiles and molecular epidemiology. Further, we offer perspectives for future surveillance approaches and reporting of AMR in this pathogen.

\section{Methods}

\subsection{Literature Search}

Between $15^{\text {th }}$ and $30^{\text {th }}$ May, 2020, the authors searched different databases (African Journals OnLine (AJOL), Scopus, MEDLINE, EBSCOhost and ISI Web of knowledge) for published literature on $S$. aureus infections in Kenya using the 
following key words: "Staphylococcus aureus", "prevalence", "antimicrobial resistance", "molecular epidemiology", "Kenya". The criteria for inclusion were: Studies reporting data from Kenya; published in English, reporting on isolates between the year 2000 and 2020, focusing on clinical $S$. aureus infections and based on a minimum of 30 non-duplicate isolates. A hand search was conducted on the bibliographic references of individual publications to identify any additional references. Further, grey literature was searched for relevant data. Abstracts of studies meeting the inclusion criteria were assessed for relevance before selection of the final dataset. Where data was lacking in the publication, attempts were made to contact the authors. In the end 18 publications were included.

\subsection{Clustering of Spa Types}

More recently, it has been suggested that spa typing combined with clustering using the based upon repeat pattern (BURP) algorithm yields results of comparable discrimination with those of multilocus sequence typing (MLST) and its associated clustering algorithm, based upon related sequence type (BURST) but with advantages of reduced costs and turnaround times [10]. As such, we retrieved sequences of all reported spa types using their repeat succession from SpaServer database (https://spa.ridom.de/index.shtml) and clustered them based on the BURP algorithm [11] using the Ridom StaphType Software (Ridom Bioinformatics). The clustering was based on the "exclude spa types with $<5$ repeats and cluster sequences if cost $\leq 4$ " parameters [11]. A total of 194 sequences classified into 61 spa types were evaluated. The spa types were distributed across four regions of Kenya namely: Nairobi (71), Thika (85), Kericho (18) and Kisu$\mathrm{mu}(20)$.

\section{Results}

\subsection{Prevalence of $S$. aureus Infections}

A majority of Kenyan studies reporting on the occurrence of $S$. aureus infections are from public and private health facilities in the capital city of Nairobi. The earliest report is by Ladhani and colleagues in the coastal county of Kilifi where bacteremia due to $S$. aureus among children aged between 1 and 12 years was observed in 5\% (106/2100) of all positive blood culture isolates between 1996 and 2001 [12]. MRSA rate was $1 \%$ of all $S$. aureus isolates. Most of these infections were community acquired with malnutrition, younger age and absence of a focus of infection identified as significant risk factors for mortality which was at the time estimated at $24 \%$ [12].

Kohli and colleagues conducted a five-year (2003-2008) retrospective analysis of records of 18,750 blood culture specimens in a private university hospital in Nairobi. A total of 1092 (5.8\%) blood stream bacterial pathogens were identified where $S$. aureus was the most frequently detected at $2 \%(368 / 18,750)$ [13]. In what may be regarded as the first report from a multi-center study, Maina et al. 
detected $S$. aureus in $45.8 \%(82 / 179)$ of specimens from skin and soft tissue infections from five centers within Nairobi (2 public hospitals, 2 private hospitals and 1 research institute) between 2005-2007 [14].

At the Moi Teaching and Referral Hospital (MTRH), Kenya's second largest national referral facility, Akoru and others recovered 107 S. aureus between 2010 and 2011 among clinical samples in nine departments including outpatient, ICU, pediatric, burn and surgical units. This study did not report on the isolation rate of $S$. aureus [15]. Rutare reported a $S$. aureus detection rate of $32.6 \%$ in $218 \mathrm{swab}$ and tracheal aspirate specimens among pediatric ICU patients at Kenyatta National Hospital in 2012 [16] while Kanaga identified S. aureus as the most common cause of wound infections at 52.6\% (79/150) among pediatric surgical patients in the same hospital in 2014 [17]. Maina et al. observed S. aureus as a leading cause of community onset infections (16\% of all Blood Stream Infections isolates) in bacteremia cases based on four-year retrospective analysis of laboratory records at Aga Khan University Hospital [18].

Aiken et al. reported $S$. aureus in $8.9 \%$ of 950 screens (733 inpatients) collected by means of repeated cross-sectional surveys in Thika Level 5 Hospital [19]. This remains the largest study evaluating carriage of MRSA among inpatients in a healthcare facility in Kenya. Nyasinga et al. detected $S$. aureus in $24.4 \%$ $(54 / 221)$ of specimens collected mainly from skin and soft tissue infections in a surveillance study covering Nairobi, Kericho and Kisumu counties between 2015 and 2016 . Over $87 \%$ of the isolates were associated with community acquired infections [9].

Reports of carriage of $S$. aureus and MRSA among healthcare workers (HCWs) in Kenya are limited. Omuse and colleagues screened 246 HCWs in a tertiary private health facility in Nairobi and isolated $S$. aureus in $18.3 \%(45 / 246)$ of them. No MRSA was isolated in the study [20]. Mogere et al. analyzed 180 nasal and hand screens at Kenyatta National Hospital and identified $S$. aureus in $34.4 \%(62 / 180)$ of HCWs out of which 59.7\% (37/62) were MRSA (Table 1) [21].

\subsection{Antimicrobial Resistance Patterns in S. aureus}

Different investigators used both manual and automated antimicrobial susceptibility testing (AST) platforms and in total AST results for 32 drugs were reported. However, for purposes of comparisons, only 11 drugs were considered as others were reported in very few studies or belonged to the same drug class. The highest proportion of MRSA was reported by Maina et al. in $84.1 \%$ of isolates from skin and soft tissue infections from five facilities within Nairobi [14]. At the Moi Teaching and Referral Hospital, Akoru et al. reported a MRSA frequency of $36.5 \%(39 / 107)$ [15]. In the same study, isolates showed $>90 \%$ resistance against erythromycin, tetracycline and rifampicin even though it is worth noting that the investigators focused on MRSA strains which are generally associated with multidrug resistance.

Kohli et al. reported a MRSA detection rate of $21 \%$ among blood stream isolates in a private hospital between 2003 and 2008 [13]. An MRSA rate of $6 \%$ and 
Table 1. A summary of reported studies on Staphylococcus aureus infections in Kenya.

\begin{tabular}{|c|c|c|c|c|c|c|c|c|c|}
\hline Year & Location & Facility & Population & $\begin{array}{l}\text { Sample } \\
\text { size }\end{array}$ & Study design & Specimen type & $\begin{array}{l}\text { S. aureus } \\
\text { detection }\end{array}$ & $\begin{array}{c}\text { MRSA } \\
\text { detection }\end{array}$ & Reference \\
\hline 1996-01 & Kilifi & Public & Children & 2100 & Retrospective & Blood & $5 \%(106 / 2100)$ & $1 \%(1 / 106)$ & [12] \\
\hline 2003-08 & Nairobi & Private & All age & 18750 & Retrospective & Blood & $2 \%(368 / 18750)$ & $21 \%(76 / 364)$ & [13] \\
\hline 2005-07 & Nairobi & $\begin{array}{c}\text { Public } \\
\text { \& private }\end{array}$ & All age & 179 & $\begin{array}{l}\text { Prospective, } \\
\text { multicenter }\end{array}$ & SSTIs & $45.8 \%(82 / 179)$ & $84.1 \%(69 / 82)$ & {$[14]$} \\
\hline 2010 & Nairobi & Private & HCWs & 246 & Prospective & Nasal swabs & $18.3 \%(45 / 246)$ & 0 & [20] \\
\hline 2011 & Thika & Public & All age & 733 & $\begin{array}{c}\text { Prospective } \\
\text { cross-sectional }\end{array}$ & Carriage swabs & $10 \%(74 / 733)$ & $7 \%(6 / 86)$ & [19] \\
\hline 2012 & Nairobi & Public & Pediatric ICU & 218 & Prospective & $\begin{array}{l}\text { Swabs \& } \\
\text { tracheal } \\
\text { aspirates }\end{array}$ & $32.6 \%(71 / 218)$ & $46.5 \%(33 / 71)$ & [16] \\
\hline 2011-13 & Nairobi & Private & All age & NR & Retrospective & Heterogeneous & $\mathrm{NR}(\mathrm{n}=731)$ & $3.7 \%(27 / 731)$ & [22] \\
\hline 2014 & Nairobi & Public & $\begin{array}{c}\text { Pediatric/ } \\
\text { Surgical }\end{array}$ & 150 & Prospective & Wounds & $\begin{array}{c}52.6 \% \\
(79 / 150)\end{array}$ & $50.6 \%(40 / 79)$ & {$[17]$} \\
\hline 2014-16 & Nairobi & Public & All age & NR & Retrospective & Heterogeneous & $\mathrm{NR}(\mathrm{n}=944)$ & $27.8 \%(232 / 831)$ & [23] \\
\hline 2014-16 & Nairobi & Public & Adults & 187 & Hybrid & Heterogeneous & NR & $53.4 \%(100 / 187)$ & [24] \\
\hline 2010-11 & Rift valley & Public & All age & NR & Retrospective & Heterogeneous & $\mathrm{NR}(\mathrm{n}=107)$ & $36.5 \%(39 / 107)$ & {$[15]$} \\
\hline 2015 & Nairobi & Public & HCWs & 180 & Prospective & $\begin{array}{c}\text { Nasal \& } \\
\text { hands swabs }\end{array}$ & $34.4 \%(62 / 180)$ & $\begin{array}{l}59.7 \% \\
37 / 62\end{array}$ & [21] \\
\hline 2015-16 & $\begin{array}{l}\text { Nairobi, } \\
\text { Kisumu \& } \\
\text { Kericho }\end{array}$ & Public & All age & 221 & Prospective & Heterogeneous & $24.4 \%(54 / 221)$ & $\begin{array}{c}11 \% \\
(6 / 54)\end{array}$ & [9] \\
\hline
\end{tabular}

HCWs-Healthcare workers; ICU-Intensive Care Unit; NR-Not reported; SSTIs-skin and soft tissue infections.

resistance of $16 \%$ and $13 \%$ against tetracycline and erythromycin respectively were reported among isolates from a private facility in Nairobi in 2014. In the same study, vancomycin and linezolid showed the highest effectiveness [18].

The lowest proportion of MRSA in Kenya was reported by Omuse and colleagues who detected MRSA strains in 3.7\% of 731 isolates from two private health facilities in Nairobi. Increased susceptibility was seen for vancomycin, teicoplanin and linezolid while $92 \%$ of the isolates were resistant to penicillin [22]. Aiken et al. showed a low MRSA detection of 7\% among inpatient carriage isolates in a public hospital outside of Nairobi while Nyasinga et al. reported an MRSA rate of $11 \%$ among isolates from three counties in Kenya [9].

Gitau et al. analyzed 944 isolates collected between 2014 and 2016 at the Kenyatta National Hospital and reported an MRSA rate of 27.8\% (262/944) (Table 2). Marked resistance against penicillin (92\%) and moderate resistance against trimethoprim/sulfamethoxazole (56.9\%) and tetracycline (33.2\%) were reported. High susceptibilities to quinupristin/dalfopristin, linezolid, teicoplanin and vancomycin were observed (95\% - 100\%) [23].

An evaluation of resistance profiles of isolates from the internal medicine department of Kenyatta National Hospital between 2014 and 2016 revealed an 
Table 2. Antimicrobial resistance profiles of Staphylococcus aureus from different studies.

\begin{tabular}{|c|c|c|c|c|c|c|c|c|c|c|c|}
\hline $\begin{array}{l}\text { Drug } \\
\text { Study }\end{array}$ & OXA/FOX & PEN & TET & TMP/SXT & ERY & CLN & GEN & CIP & RIF & LZD & VAN \\
\hline $\begin{array}{c}{[13]} \\
(\mathrm{n}=364)\end{array}$ & $21 \%$ & NR & NR & $19 \%$ & $35 \%$ & NR & $0.8 \%$ & $18 \%$ & NR & NR & $0.5 \%$ \\
\hline $\begin{array}{c}{[19]} \\
(\mathrm{n}=86)\end{array}$ & $7 \%$ & $75.6 \%$ & $21 \%$ & $44 \%$ & NR & $2 \%$ & $8 \%$ & $7 \%$ & $7 \%$ & NR & NR \\
\hline $\begin{array}{c}{[14]} \\
(\mathrm{n}=82)\end{array}$ & $84.1 \%$ & NR & NR & $75.6 \%$ & $81.7 \%$ & $37.8 \%$ & $84.1 \%$ & $74.4 \%$ & NR & NR & 0 \\
\hline $\begin{array}{c}{[16]} \\
(\mathrm{n}=71)\end{array}$ & $46.5 \%$ & NR & $29.6 \%$ & $45.5 \%$ & $47.9 \%$ & $8.5 \%$ & $38 \%$ & $36.6 \%$ & NR & $1.4 \%$ & $1.4 \%$ \\
\hline $\begin{array}{c}{[22]} \\
(\mathrm{n}=731)\end{array}$ & $3.7 \%$ & $92.2 \%$ & $15.5 \%$ & $42.1 \%$ & $11.9 \%$ & $10 \%$ & $1.9 \%$ & NR & $0.4 \%$ & NR & NR \\
\hline $\begin{array}{c}{[15]} \\
(\mathrm{n}=39)^{\star}\end{array}$ & $36.5 \%$ & NR & $92 \%$ & NR & $92 \%$ & $87 \%$ & $77 \%$ & $72 \%$ & $90 \%$ & $23 \%$ & $26 \%$ \\
\hline $\begin{array}{c}{[23]} \\
(\mathrm{n}=944)\end{array}$ & $28 \%$ & $92 \%$ & $33 \%$ & $57 \%$ & $26 \%$ & $7 \%$ & $13 \%$ & NR & $8 \%$ & $3 \%$ & $5 \%$ \\
\hline $\begin{array}{c}{[21]} \\
(\mathrm{n}=62)\end{array}$ & $18.9 \%$ & $66.1 \%$ & $35.5 \%$ & $46.8 \%$ & $51.6 \%$ & NR & $3.2 \%$ & $22.6 \%$ & NR & $1.6 \%$ & $53.2 \%^{* *}$ \\
\hline $\begin{array}{c}{[9]} \\
(\mathrm{n}=52)\end{array}$ & $11 \%$ & $98 \%$ & $21 \%$ & $67 \%$ & $17 \%$ & $13 \%$ & $15 \%$ & $13.5 \%$ & $11.6 \%$ & 0 & 0 \\
\hline $\begin{array}{c}{[25]} \\
(\mathrm{n}=32)\end{array}$ & $21 \%$ & $100 \%$ & $34.3 \%$ & $40.6 \%$ & $34.3 \%$ & $28 \%$ & $25 \%$ & NR & $37.5 \%$ & 0 & 0 \\
\hline
\end{tabular}

OXA: Oxacillin, FOX: Cefoxitin, PEN: Penicillin, TET: Tetracycline, TMP/SXT: Trimethoprim/sulfamethoxazole, ERY: Erythromycin, CLN: Clindamycin, GEN: Gentamycin, CIP: Ciprofloxacin, RIF: Rifampicin, LZD: Linezolid, VAN: Vancomycin. ${ }^{*}$ The resistance values reported are based on MRSA strains. **Susceptibility to vancomycin was tested using disc diffusion method. NR: Not reported.

MRSA detection rate of $53.5 \%$ [24] while K'yanya et al. recently analyzed the susceptibility profiles of 32 clinical isolates from four hospitals across Kenya where higher susceptibilities to vancomycin, teicoplanin, tigecycline and linezolid were observed [25]. However, there were decreased susceptibilities to trimethoprim-sulfamethoxazole, tetracycline and ciprofloxacin and complete resistance to penicillin [25]. The authors applied whole genome sequencing (WGS) to uncover both known and novel mechanisms of resistance which marked the first Kenyan study employing genomic approaches to characterize the genetic basis of resistance in clinical staphylococcal isolates [25].

Overall, the resistance ranges for seven most commonly tested drugs were: tetracycline $(15.5 \%-35.5 \%)$, trimethoprim-sulfamethoxazole (19\% - 75.6\%), erythromycin $(11.9 \%-81.7 \%)$, clindamycin $(2 \%-37.8 \%)$, gentamicin $(1 \%-84.1 \%)$ and ciprofloxacin $(7 \%-74.4 \%)$. A major observation was the diminished efficacy of penicillin which varied from $66 \%$ in [21] to $100 \%$ in [25].

\subsection{Molecular Characterization}

The first report on staphylococcal cassette chromosome mec (SCCmec) charac- 
terization of MRSA was by Maina et al. who reported SCCmec II among MRSA strains from SSTIs in Nairobi [14]. MRSA strains circulating in Thika level 5 hospital over a four-month period were classified as SCCmec III [19] as were isolates from Aga Khan University Hospital and one public health facility in Nairobi where SCCmec type-III [3A] predominated followed by SCCmec-IV [2B] [26].

Omuse et al. assessed the detection frequencies of the Panton-Valentine leucocidin ( $p v l)$ gene among 45 carriage and 63 infective isolates from a private health facility in Nairobi. $P_{V} l$ was identified in $24.4 \%$ and $39.7 \%$ of carriage and infective isolates respectively even though the differences did not attain statistical significance [27]. In the same study, there were significant differences in the occurrence of $p v l$ between skin and soft tissue infections isolates and isolates from other sources [27]. Maina et al. reported $p v l$ at a frequency of $20.3 \%$ among MRSA isolates [14] while among carriage isolates recovered from inpatients at Thika Level 5 Hospital it was 17\% [19]. Kyany'a et al. recently applied whole genome sequencing (WGS) to identify multiple virulence genes and correlated their occurrence with markers of severity of infection on a background of clonality where a handful showed significant associations [25].

Spa and MLST typing schemes have emerged as the most frequently used molecular methods for evaluating the evolutionary history of $S$. aureus [10]. The first report on molecular characterization of MRSA was by Aiken et al. who employed DNA microarrays, spa and MLST typing. The study identified 28 unique spa types (20 STs) in a collection of $86 \mathrm{~S}$. aureus isolates where predominant strains included ST22 (t223), ST8 (t064), ST239 (t037) and ST1290 (t131). All MRSA strains belonged to ST239 ( $\mathrm{t} 037$ ). Overall, the strains were clustered into 14 MLST clonal complexes which included CC1, CC5, CC8, CC15, CC22, CC30, CC45, CC121 and CC239. Several spa types were represented by individual isolates which reflected the heterogeneity in the circulating MSSA strains. Five novel spa types were described and were all represented by MSSA strains. While two novel spa types belonged to common STs (ST8 and 22), three corresponded to STs that clustered as singletons [19].

An analysis of 93 isolates from a private and public hospital in Nairobi by pulsed field gel electrophoresis (PFGE), spa and MLST identified 19 PFGE types and 12 singletons which corresponded to 40 spa types (18 MRSA and 22 MSSA). Global MRSA strain ST241 (t037) was identified as the predominant strain. Five novel spa types including two MRSA strains (t13149/t13150) were also identified [26]. In the same study, MLST CC5 which included STs 1, 5, 8 and 241 predominated [26].

Nyasinga et al. identified 22 spa types (21 STs) among isolates from three counties (Nairobi, Kericho and Kisumu) where t355 (ST152) and t037 (ST241) were the dominant MSSA and MRSA strains respectively. In addition, two novel MSSA spa types were identified. Many spa/ ST types were represented by single isolates and showed unique association with each region [9]. Kyany'a et al. uti- 
lized WGS to characterize the strain diversity of clinical isolates from different hospitals. Clonal classifications yielded 12 STs and 15 spa types that were clustered into 12 clonal complexes which included the global groups such as CC5, CC8, CC22, CC152 [25].

BURP clustering included 191 isolates (59 spa types) after excluding 2 spa types (t4198 and t10499) representing 3 isolates. Of these, 42 were MRSA while 152 were MSSA. t037 was the most common strain overall and consequently, the most dominant MRSA strain, accounting for over $45 \%$ of all MRSA strains. Other common strains included t064, t355, t223, t131 and t005. MRSA strains were represented by 16 spa types while MSSA strains were represented by $41 s p a$ types. Four spa types contained both MRSA and MSSA strains. The algorithm clustered isolates into 12 clusters and 19 singletons. However, four clusters including spa CC359, spa CC005, spa CC121 and spa CC021 accounted for 59\% (113/194) of all isolates. The four dominant clusters were composed of between 5 and 7 spa types each with seven of the remaining 8 clusters represented by two spa types each (Figure 1).

In terms of regional distribution, 49 spa types were associated with individual regions while only one spa type (t355) was present in all the four regions (Figure 2). Three of the four dominant clusters had representative spa types from three regions while one had spa types from all four regions. There was marked heterogeneity among strains from Nairobi with overlaps with Thika and Kisumu and to a lesser extent, Kericho. Fewer strains were uniquely associated with Kisumu and Kericho regions even though this may be explained by lower isolate representation from the regions.
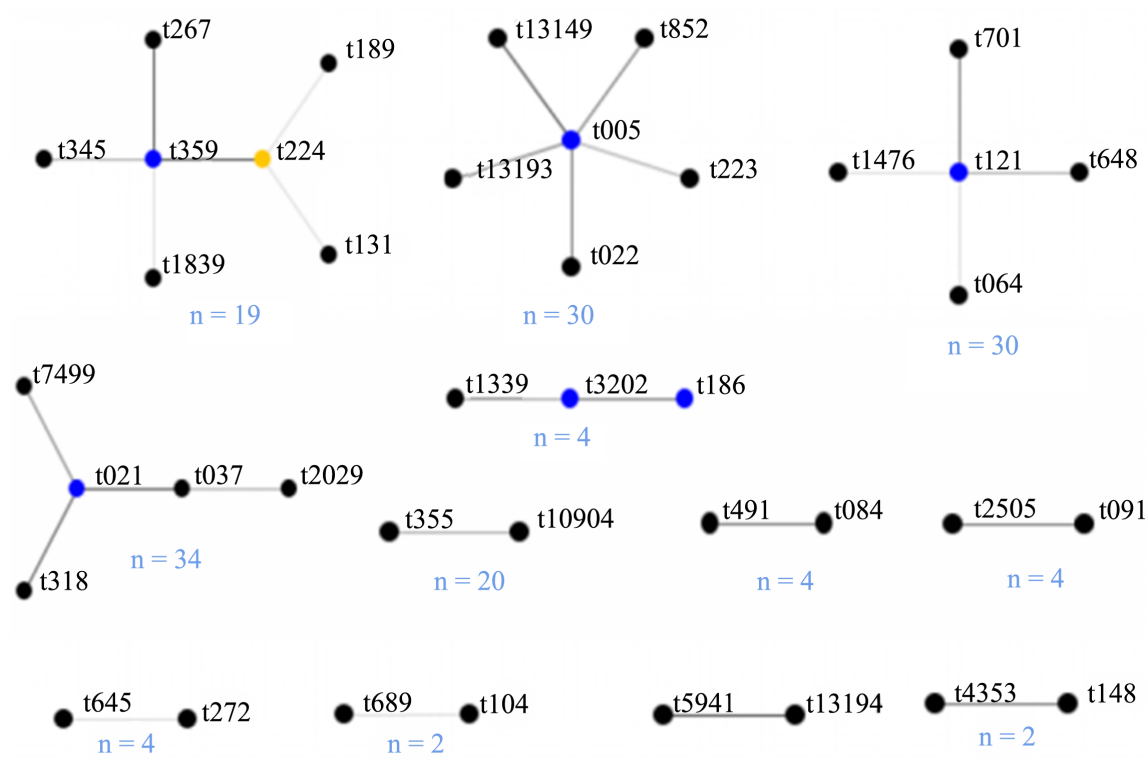

Figure 1. A based upon repeat pattern clustering analysis of spa types from different regions of Kenya. A total of 40 spa types were clustered into 12 clusters while 19 spa types were classified as singletons. Two spa types (t4198 and t10499) were excluded in the analyses. Blue circles indicate the founders of each cluster. 


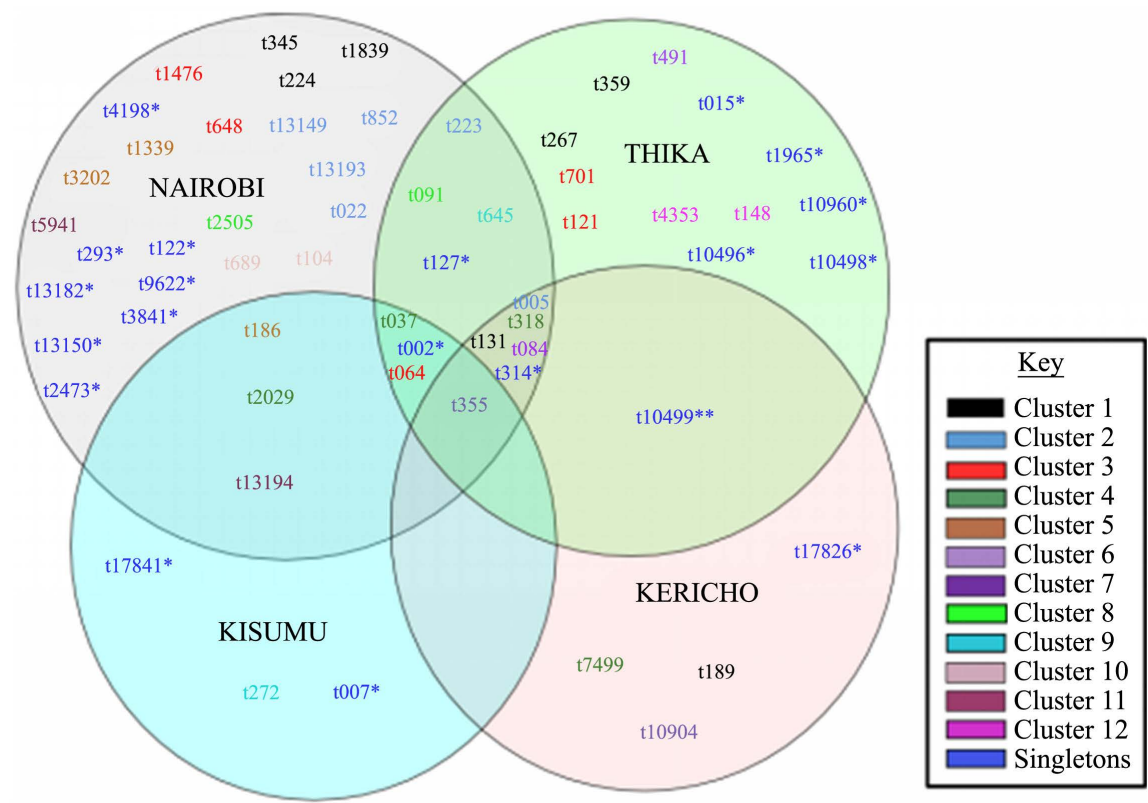

Figure 2. A Venn diagram showing the distribution of reported spa types across four regions of Kenya. Different clusters and singletons are represented by different colors. Spa types t021 and t3772 (not displayed) occurred in Nairobi and Kericho and Thika and Kisumu respectively. ${ }^{\star}$ Singletons; ${ }^{* *}$ spa types excluded from clustering analyses.

\section{Discussion}

\subsection{Isolation of $S$. aureus}

Examination of available data showed variable rates of clinical $S$. aureus detection in Kenya (2\% - 52.6\%) with a majority of studies skewed in favor of Nairobi. Reported $S$. aureus isolation rates from other African countries have been inconsistent. In Eritrea, Garoy and others detected $S$. aureus in $63.1 \%$ of 130 specimens, mostly pus from two referral hospitals in Asmara [28]. S. aureus was detected in $28.7 \%$ of surgical site infections in Uganda [29] while in Tanzania, the prevalence of $S$. aureus infections has been estimated to vary from $1 \%-60 \%$ [30]. The variable detection of $S$. aureus may be attributable to differences in characteristics of specific study populations, the type of specimens collected, type of healthcare facility and methodological differences. For instance, the bulk of isolates were recovered from skin and soft tissue infection specimens whereas blood cultures and other specimens had lower isolation frequencies. Further, private health facilities had lower isolation frequencies compared to public health facilities, probably due to effective implementation of infection prevention and control (IPC) programs and differences in the socio-demographic profiles of patients visiting such facilities compared to public ones. Some of these factors have been attributed to variable isolation and AMR rates in other African countries [5] [28].

Identification of $S$. aureus in most hospital laboratories in Kenya is based on growth characteristics, gram stain and biochemical properties [22]. Specifically, the coagulase test plays a critical role in distinguishing $S$. aureus from coagulase 
negative staphylococci (CoNS). CoNS are more ubiquitous and may co-habit with $S$. aureus on the skin and the anterior nares and thus their misidentification as $S$. aureus would erroneously overestimate the burden of $S$. aureus in a given setting [24]. Most CoNS are resistant to methicillin and other drugs and their testing would exaggerate MRSA rates, further emphasizing the importance of accurate identification.

In recent years, research institutes, major public and most private health facilities have adopted the use of automated bacterial identification platforms such as the VITEK system [6] [22]. Despite their higher financial costs in comparison to traditional identification, such platforms would increase the accuracy of identification. Matrix Assisted Laser Desorption/Ionization-Time of Flight Mass Spectrometry (MALDI-TOF MS) is another technology whose utility in clinical diagnosis is beginning to be embraced albeit slowly due to its prohibitive costs in resource-limited settings [31].

Many studies did not distinguish between community and hospital acquired infections. However, Kyany'a et al. and Nyasinga et al. reported such delineation where the bulk of skin and soft tissue infections caused by $S$. aureus were community acquired [9] [25]. The distinction between community and healthcare associated infections is important especially with regards to MRSA strains which display multidrug resistance. Community acquired MRSA (CA-MRSA) strains are associated with possession of smaller mobile genetic elements (SCCmec) that contain fewer resistance genes and hence display limited multidrug resistance compared to healthcare associated MRSA strains (HA-MRSA) that contain larger elements with additional resistance determinants [3]. Further, CA-MRSA strains have been suggested as being more virulent compared to HA-MRSA with the possession of the Panton-Valentine leucocidin and acquisition of arginine catabolic mobile element (ACME) [32]. However, clinically distinguishing HA-MRSA from CA-MRSA is challenging as community acquired strains may initiate infections in the healthcare setting while healthcare associated strains may be transferred to the community where they can cause infections [8] [33].

Only two studies have evaluated $S$. aureus and MRSA carriage among HCWs. One was performed in a public health facility where $S$. aureus detection was $34 \%$ with an MRSA proportion of 59\%. The other study was conducted in a private hospital where $S$. aureus was identified in $18 \%$ of HCWs with none being MRSA. A Cameroonian study reported a detection frequency of $40.6 \%$ among HCWs and inpatients, which was higher than the highest reported rate from the two Kenyan studies [34]. In the neighboring country of Tanzania, S. aureus was detected in $41.4 \%$ of HCWs in tertiary hospitals out of which $37.5 \%$ were MRSA [35]. In Ethiopia, $S$. aureus detection among healthcare workers from two hospitals was $12 \%$ with an MRSA rate of $48.3 \%$ [36] while in Uganda, $S$. aureus detection and MRSA proportion were $28.8 \%$ and $46.4 \%$ respectively [37]. In contrast to African studies, a pooled MRSA detection of $1.8 \%$ has been reported among HCWs in Europe and the USA [38]. MRSA carriage among HCWs poses challenges in infection prevention and control prevention measures as colonized 
individuals may act as reservoirs of infection for vulnerable patients. Transmission may occur even when colonized individuals are asymptomatic, though these professionals likely act as vectors rather than direct transmitters [39]. Since MRSA colonization is a risk factor for infection, infections may occur among HCWs. Limited data exists on inpatient carriage of MRSA infections in Kenya as only one study has previously explored this phenomenon thus revealing a gap for future inquiry.

Given the skewed distribution of studies towards Nairobi and specifically the Kenyatta National Hospital, it is difficult at present to establish a national estimate of the prevalence of staphylococcal infections. In many studies, data was analyzed retrospectively and inevitably, there were gaps in clinical and socio-demographic data which may be useful in the estimation of risk factors for infection or AMR spread. However, data from prospective, multicenter studies covering other areas of the country are increasingly being reported [9] [25]. Further, the establishment of a national surveillance and reporting framework will enable more accurate estimations of the extent of infections caused by $S$. aureus.

\subsection{Antimicrobial Resistance Profiles of $S$. aureus}

Reported MRSA detection varied from as low as $1 \%$ in blood isolates to as high as $84.1 \%$ in skin and soft tissue infection isolates. Estimated MRSA detection rates from other African countries include Tunisia (12\% - 46\%), Libya (31\%), South Africa (23\% - 39\%), Algeria (35\% - 75\%), Botswana (23\% - 44\%), Morocco $(23 \%)$, Egypt (52\% - 82\%), Nigeria (9\% - 41\%), Madagascar $(6 \%)$ and Ethiopia (55\%) [5]. In Tanzania, MRSA prevalence has recently been reported at $33 \%$, up from an earlier estimate of $16 \%$ [30]. A study from Eritrea reported an MRSA proportion of $72 \%$ [28] while in Uganda, an MRSA rate of $31.5 \%$ among surgical site infections was estimated [29]. Gonsu and others observed an MRSA rate of $85 \%$ among Cameroonian HCWs and hospitalized patients which was higher than the highest recorded rates in Kenya among HCWs (59.7\%) and inpatients (7\%) [34]. The variable rates of MRSA and resistance to other drugs has been previously attributable to methodological variations, differences in antibiotic use, patient characteristics, variable HIV and TB burden in specific settings and whether sampling is performed on rural or urban populations [5] [24].

Resistance to other drugs may be assessed in three categories: Complete, moderate to high or negligible resistance. Penicillin was consistently reported as having lost efficacy with most studies showing resistance rates between $92 \%$ $100 \%$. Moderate to high resistance was reported in tetracycline $(15.5 \%-35.5 \%)$, trimethoprim-sulfamethoxazole (19\% - 75.6\%); erythromycin $(11.9 \%-81.7 \%)$; clindamycin (2\% - 37.8\%); gentamicin ( $1 \%-84.1 \%)$, ciprofloxacin $(7 \%-74.4 \%)$ and rifampicin $(0.5 \%-37.5 \%)$. In Tanzania, resistance against penicillin, erythromycin, trimethoprim-sulfamethoxazole and tetracycline has been observed at $85 \%, 49 \%, 38 \%$ and $28 \%$ respectively [30]. Garoy et al. recently observed resistance rates of $1 \%, 11 \%$ and $15.9 \%$ against gentamicin, erythromycin and vanco- 
mycin [28]. However, the authors tested susceptibility to vancomycin using the disc diffusion method which is not reliable in inferring resistance against the drug. Besides penicillin whose efficacy has waned over the years, there seems to be an increasing trend of reduced susceptibility to trimethoprim-sulfamethoxazole (TMP-SXT), erythromycin and tetracycline. Resistance against these drugs is primarily mediated by the $d f$, erm and tet genes respectively. The high burden of HIV/AIDS in Africa where TMP-SXT is widely used for patient management has been cited as a potential driver of widespread resistance due to increased selection pressure [25]. Further, widespread veterinary use of antibiotics such as tetracycline coupled with weak regulatory systems contributes to emergence of resistance.

Mupirocin is an important agent for treatment of skin and soft tissue infections but also for topical decolonization of patients and healthcare workers. However, mupirocin resistant $S$. aureus (MupRSA) strains are increasingly being reported [40]. Resistance may be mediated by point mutations or horizontal acquisition of plasmids carrying resistance genes such as mupA and mupB [41]. Mupirocin resistance in Kenya as reported by the few studies ranged from $0 \%$ $18 \%$ in comparison with the continental pooled estimate of $14 \%(0 \%-49.6 \%)$ [40]. High level mupirocin resistance has recently been observed among carriage strains in Uganda [42].

Last line treatments such as vancomycin, linezolid, daptomycin and teicoplanin had negligible to no resistance $(<5 \%)$. At the continental level comparable rates have been reported with MRSA strains showing greater resistance [5]. Linezolid, an oxazolidinone that acts by binding to domain $\mathrm{V}$ of the $23 \mathrm{~S}$ ribosomal sub-unit to inhibit functional ribosomal complex assembly and daptomycin, a lipopeptide that acts on the cell membrane in a calcium ion dependent manner are highly effective against MRSA strains. However, resistance against linezolid, mediated by point mutations and the plasmid-borne chloramphenicol florfenicol resistance (cfr) gene has already been observed in other settings [43]. The cfr gene has also been implicated in phenotypic expression of resistance against other classes such as lincosamides and phenicols [44]. Resistance against daptomycin, mediated by a variety of mechanisms is increasingly being recognized in $S$. aureus [45]. Initial observation of resistance coupled with the expiry of patents for the two drugs and their impending decreased costs and greater availability calls for vigilance through constant surveillance for emerging resistant strains as prolonged use has been suggested as a key driver of linezolid resistance. The threat of widespread resistance is compounded by the occurrence of linezolid and high level daptomycin resistance among coagulase negative Staphylococci [44] which may act as a pool of resistance genes for the more pathogenic $S$. aureus [46].

There is a need for uniformity in interpretation and reporting of isolate susceptibilities to different drug classes in accordance with international guidelines such as the CLSI. For example, there were instances where susceptibility to vancomycin was tested using disc diffusion method and was not verified by other methods such as minimum inhibitory concentration (MIC) determination or mo- 
lecular detection of vancomycin resistance genes. Incorporation of molecular methods such as PCR and whole genome sequencing in routine AMR surveillance would reveal existing and novel mechanisms of resistance. Further, characterization of resistance among CoNS would uncover potential transmission pathways of existing and new resistance mechanisms.

\subsection{Molecular Characterization of Staphylococcus aureus}

The Panton-Valentine leucocidin, a bi-component exotoxin encoded by LukSand $L u k F-P V$ genes that acts by forming pores on target immune cells was reported at frequencies of between $17 \%$ and $33 \%$. A Gambian study established a PVL prevalence of $61.4 \%$ among bacteremia and SSTI isolates [47]. At the continental level, PVL prevalence has varied from $0.3 \%-100 \%$ [8]. The role of PVL in mediating severity of infection has been controversial with some studies linking it to syndromes such as $S$. aureus mediated pyomyositis and necrotizing pneumonia [48] but not others [49]. The prevalence of the toxin seems to be significantly higher in Africa compared to other regions and investigations on its higher prevalence and potential role in disease pathogenesis are invited.

BURP clustering revealed the presence of four major clusters: CC359, CC005, CC121 and CC021. Nairobi region showed marked heterogeneity with representatives from different clusters as well as singletons. Further, strains from Nairobi showed considerable overlap with strains from other regions (Figure 2). This heterogeneity may be explained by the population size of Nairobi city county which is the most populous in the country coupled with increased human mobility into and out of the city. Overall, spa types t037, t064, t355 and t223 were dominant. There seems to be limited heterogeneity among MRSA strains with ST239/241 (t037) being the dominant strain. In Uganda, t645, t4353, t355, t037 and t064 are the most common with t645 and t4353 appearing less frequently in Kenya [42]. Spa types t037 has been associated with HA-MRSA strains while t064 has been linked to CA-MRSA infections in Uganda [42]. Spa types t042 and t044 have been observed in Egypt and Algeria and were not reported in Kenya [8] whereas in Ghana, t355, t084, t10519 and t008 have been the preponderant strains [50]. Contrary to Kenya where ST239 (t037), ST152 (t355) and ST8 (t064) are dominant, ST88 which is the quintessential African strain has been suggested as the predominant circulating strain in Tanzania [30].

From the collection evaluated, CA-MRSA strain USA3OO (ST 8/CC8; t008/spa CC008) was not observed. However, t002 (ST5) which is associated with HA-MRSA infections (USA1OO) was observed in Nairobi, Thika and Kisumu though none of the isolates were MRSA. The observation of pandemic ST239/241(t037) strain in circulation calls for increased surveillance to track transmission of MRSA strains in Kenya. Further, molecular characterization is essential to unravel the changing epidemiology of the pathogen where increased human travel across borders and other factors may lead to displacement of existing clones by introduction of fitter ones as happened in the United Kingdom where EMRSA-15 (ST22) and EMRSA-16 (ST36) displaced other MRSA strains 
to establish their dominance [51].

\section{Concluding Remarks}

Currently available data points to $S$. aureus variable detection and AMR rates in Kenya. While penicillin has lost its efficacy, there are hints of decreasing effectiveness for commonly prescribed antimicrobial classes. Many of the last line and recent treatments remain largely effective. The population structure of $S$. aureus in Kenya is heterogeneous with the presence of major global lineages in circulation.

Presently, it is difficult to establish the clinical and economic burden of MRSA infections in Kenya due to factors such as weak national AMR surveillance and reporting mechanisms, self-medication and poor regulation of antimicrobial use. Further, inadequate diagnostic microbiology infrastructure in many health facilities coupled with empirical treatments are major obstacles [52]. In light of this, the Kenya government enacted the national policy for prevention and containment of AMR which recognizes the threat of AMR and seeks to initiate and implement AMR prevention and control strategies in line with the global action plan (GAP) on tackling AMR from a One Health perspective.

Antimicrobial resistance remains a One Health challenge that warrants interdisciplinary efforts at the human-animal-environment interface. However, there remains limited data on the prevalence of drug resistant staphylococcal infections among animal populations and environmental settings in Kenya, an area where collaborative efforts are imperative.

\section{Acknowledgements}

This work was supported by the German Research Foundation (DFG) (https://www.dfg.de/en/) under the Staphylococci in Africa-Resistance and Epidemiology (ShARE) grant to GR. The funders had no role in study design, data collection and analysis, decision to publish or preparation of the manuscript.

\section{Conflicts of Interest}

The authors declare no conflicts of interest regarding the publication of this paper.

\section{References}

[1] Tong, S.Y., Davis, J.S., Eichenberger, E., Holland, T.L. and Fowler, V.G. (2015) Staphylococcus aureus Infections: Epidemiology, Pathophysiology, Clinical Manifestations, and Management. Clinical Microbiology Reviews, 28, 603-661. https://doi.org/10.1128/CMR.00134-14

[2] World Health Organization (2014). Antimicrobial Resistance: Global Report on Surveillance. World Health Organization, Geneva.

[3] DeLeo, F.R., Otto, M., Kreiswirth, B.N. and Chambers, H.F. (2010) Community-Associated Meticillin-Resistant Staphylococcus aureus. Lancet, 375, 1557-1568. https://doi.org/10.1016/S0140-6736(09)61999-1

[4] Parker, D. (2018) A Live Vaccine to Staphylococcus aureus Infection. Virulence, 9, 
700-702. https://doi.org/10.1080/21505594.2018.1426965

[5] Falagas, M.E., Karageorgopoulos, D.E., Leptidis, J. and Korbila, I.P. (2013) MRSA in Africa: Filling the Global Map of Antimicrobial Resistance. PLOS ONE, 8, e68024. https://doi.org/10.1371/journal.pone.0068024

[6] Wangai, F.K., Masika, M.M., Lule, G.N., Karari, E.M., Maritim, M.C., Jaoko, W.G., et al. (2019) Bridging Antimicrobial Resistance Knowledge Gaps: The East African Perspective on a Global Problem. PLOS ONE, 14, e0212131. https://doi.org/10.1371/journal.pone.0212131

[7] Founou, R.C., Founou, L.L. and Essack, S.Y. (2017) Clinical and Economic Impact of Antibiotic Resistance in Developing Countries: A Systematic Review and Metaanalysis. PLoS ONE, 12, e0189621. https://doi.org/10.1371/journal.pone.0189621

[8] Abdulgader, S.M., Shittu, A.O., Nicol, M.P. and Kaba M. (2015) Molecular Epidemiology of Methicillin-Resistant Staphylococcus aureus in Africa: A Systematic Review. Frontiers in Microbiology, 6, 348. https://doi.org/10.3389/fmicb.2015.00348

[9] Nyasinga, J., Kyany'a, C., Okoth, R., Oundo, V., Matano, D., Wacira, S., et al. (2019) A Six-Member SNP Assay on the IPlex MassARRAY Platform Provides a Rapid and Affordable Alternative for Typing Major African Staphylococcus aureus Types. Access Microbiology, 1, e000018. https://doi.org/10.1099/acmi.0.000018

[10] O’Hara, F.P., Suaya, J.A., Ray, G.T., Baxter, R., Brown, M.L., Mera, R.M., et al. (2016) Spa Typing and Multilocus Sequence Typing Show Comparable Performance in a Macroepidemiologic Study of Staphylococcus aureus in the United States. Microbial Drug Resistance, 22, 88-96. https://doi.org/10.1089/mdr.2014.0238

[11] Mellmann, A., Weniger, T., Berssenbrügge, C., Rothgänger, J., Sammeth, M., Stoye, J., et al. (2007) Based upon Repeat Pattern (BURP): An Algorithm to Characterize the Long-term Evolution of Staphylococcus aureus Populations Based on Spa Polymorphisms. BMC Microbiology, 7, Article No. 98. https://doi.org/10.1186/1471-2180-7-98

[12] Ladhani, S., Konana, O., Mwarumba, S. and English, M. (2004) Bacteraemia Due to Staphylococcus aureus. Archives of Disease in Children, 89, 568-571. https://doi.org/10.1136/adc.2003.026781

[13] Kohli, R., Omuse, G. and Revathi, G. (2010) Antibacterial Susceptibility Patterns of Blood Stream Isolates in Patients Investigated at the Aga Khan University Hospital, Nairobi. East African Medical Journal, 87, 74-80. https://doi.org/10.4314/eamj.v87i2.60592

[14] Maina, E.K., Kiiyukia, C., Wamae, C.N., Waiyaki, P.G. and Kariuki, S. (2013) Characterization of Methicillin-Resistant Staphylococcus aureus from Skin and Soft Tissue Infections in Patients in Nairobi, Kenya. International Journal of Infectious Diseases, 17, E115-E119. https://doi.org/10.1016/j.ijid.2012.09.006

[15] Akoru, C., Kuremu, R.T., Ndege, S.K., Obala, A., Smith, J.W. and Bartlett, M. (2016) Prevalence and Antimicrobial Susceptibility of Methicillin Resistant Staphylococcus aureus at Moi Teaching and Referral Hospital Eldoret. Open Journal Medical Microbiology, 6, 9-16. https://doi.org/10.4236/ojmm.2016.61003

[16] Rutare, S. (2013) Prevalence of Methicillin Resistant Staphylococcus aureus (MRSA) among Paediatric Patients Admitted in Intensive Care Unit and Neonatal Intensive Care Unit at Kenyatta National Hospital-Nairobi. University of Nairobi, Nairobi.

[17] Kanaga, E.L. (2014) Antimicrobial Susceptibility of Bacteria that Cause Wound Sepsis in the Paediatric Surgical Patients at Kenyatta National Hospital. University of Nairobi, Nairobi.

[18] Maina, D., Omuse, G. and Gunturu, R. (2016) Spectrum of Microbial Diseases and 
Resistance Patterns at a Private Teaching Hospital in Kenya: Implications for Clinical Practice. PLoS ONE, 11, e0147659.

https://doi.org/10.1371/journal.pone.0147659

[19] Aiken, A.M., Mutuku, I.M., Sabat, A.J., Akkerboom, V., Mwangi, J., Scott, J.A.G., et al. (2014) Carriage of Staphylococcus aureus in Thika Level 5 Hospital, Kenya. A Cross-Sectional Study, 3, Article No. 22. https://doi.org/10.1186/2047-2994-3-22

[20] Omuse, G., Kariuki, S. and Revathi, G (2012) Unexpected Absence of Meticillin-Resistant Staphylococcus aureus Nasal Carriage by Healthcare Workers in a Tertiary Hospital in Kenya. Journal of Hospital Infection, 80, 71-73. https://doi.org/10.1016/j.jhin.2011.09.009

[21] Mogere, A. (2015) Carriage Rate of Methicillin-Resistant Staphylococcus aureus among Healthcare Workers at the Kenyatta National Hospital. University of Nairobi, Nairobi.

[22] Omuse, G., Kabera, B. and Revathi, G. (2014) Low Prevalence of Methicillin Resistant Staphylococcus aureus as Determined by an Automated Identification System in Two Private Hospitals in Nairobi, Kenya: A Cross-Sectional Study. BMC Infectious Diseases, 14, Article No. 669. https://doi.org/10.1186/s12879-014-0669-y

[23] Gitau, W., Masika, M., Musyoki, M., Museve, B. and Mutwiri, T. (2018) Antimicrobial Susceptibility Pattern of Staphylococcus aureus Isolates from Clinical Specimens at Kenyatta National Hospital. BMC Research Notes, 11, Article No. 226. https://doi.org/10.1186/s13104-018-3337-2

[24] Wangai, F.K., Masika, M.M., Maritim, M.C. and Seaton, R.A. (2019) MethicillinResistant Staphylococcus aureus (MRSA) in East Africa: Red Alert or Red Herring? BMC Infectious Diseases, 19, Article No. 596.

https://doi.org/10.1186/s12879-019-4245-3

[25] Kyany'a, C., Nyasinga, J., Matano, D., Oundo, V., Wacira, S., Sang, W., et al. (2019) Phenotypic and Genotypic Characterization of Clinical Staphylococcus aureus Isolates from Kenya. BMC Microbiology, 19, Article No. 245. https://doi.org/10.1186/s12866-019-1597-1

[26] Omuse, G., Van Zyl, K.N., Hoek, K., Abdulgader, S., Kariuki, S., Whitelaw, A., et al. (2016) Molecular Characterization of Staphylococcus aureus Isolates from Various Healthcare Institutions in Nairobi, Kenya: A Cross Sectional Study. Annals of Clinical Microbiology and Antimicrobials, 15, Article No. 51. https://doi.org/10.1186/s12941-016-0171-Z

[27] Omuse, G., Shivachi, P., Kariuki, S. and Revathi, G. (2013) Prevalence of Panton Valentine Leukocidin in Carriage and Infective Strains of Staphylococcus aureus at a Referral Hospital in Kenya. Open Journal of Medical Microbiology, 3, 5-11. http://dx.doi.org/10.4236/ojmm.2013.31002

[28] Garoy, E.Y., Gebreab, Y.B., Achila, O.O., Tekeste, D.G., Kesete, R., Ghirmay, R., et al. (2019) Methicillin-Resistant Staphylococcus aureus (MRSA): Prevalence and Antimicrobial Sensitivity Pattern among Patients-A Multicenter Study in Asmara, Eritrea. Canadian Journal of Infectious Diseases and Medical Microbiology. 2019, Article ID: 8321834. https://doi.org/10.1155/2019/8321834

[29] Ojulong, J., Mwambu, T., Jolobo, M., Agwu, E., Bwanga, F., Najjuka, C., et al. (2008) Prevalence of Methicillin Resistant Staphylococcus aureus (MRSA) among Isolates from Surgical Site Infections in Mulago Hospital-Kampala, Uganda. Internet Journal of Infectious Diseases, 7, 34-45.

[30] Mzee, T., Kazimoto, T., Madata, J., Masalu, R., Bischoff, M., Matee, M., et al. (2020) Prevalence, Antimicrobial Susceptibility and Genotypic Characteristics of Staphy- 
lococcus aureus in Tanzania: A Systematic Review. arXiv: rs.3.rs-16889/v1. https://doi.org/10.21203/rs.3.rs-16889/v1

https://www.researchgate.net/publication/340198296 Prevalence antimicrobial sus ceptibili-

ty and genotypic characteristics of Staphylococcus aureus in Tanzania a syste matic review

[31] Singhal, N., Kumar, M., Kanaujia, P.K. and Virdi, J.S. (2015) MALDI-TOF Mass Spectrometry: An Emerging Technology for Microbial Identification and Diagnosis. Frontiers in Microbiology, 6, 791. https://doi.org/10.3389/fmicb.2015.00791

[32] Shore, A.C., Rossney, A.S., Brennan, O.M., Kinnevey, P.M., Humphreys, H., Sullivan, D.J., et al. (2011) Characterization of a Novel Arginine Catabolic Mobile Element (ACME) and Staphylococcal Chromosomal Cassette mec Composite Island with Significant Homology to Staphylococcus epidermidis ACME Type II in MethicillinResistant Staphylococcus aureus Genotype ST22-MRSA-IV. Antimicrobial Agents and Chemotherapy, 55, 1896-1905. https://doi.org/10.1128/AAC.01756-10

[33] Kateete, D.P., Bwanga, F., Seni, J., Mayanja, R., Kigozi, E., Mujuni, B., et al. (2019) CA-MRSA and HA-MRSA Coexist in Community and Hospital Settings in Uganda. Antimicrobial Resistance \& Infection Control, 8, Article No. 94. https://doi.org/10.1186/s13756-019-0551-1

[34] Gonsu, K., Kouemo, S., Toukam, M., Ndze, V. and Koulla, S. (2013) Nasal Carriage of Methicillin Resistant Staphylococcus aureus and its Antibiotic Susceptibility Pattern in Adult Hospitalized Patients and Medical Staff in Some Hospitals in Cameroon. Journal of Microbiology and Antimicrobials, 5, 29-33. https://doi.org/10.5897/JMA2012.0232

[35] Moyo, S.J., Nkinda, L., Majigo, M., Rugarabamu, S., Mkashabani, E.G., Mmbaga, E.J., et al. (2018) Nasal Carriage of Methicillin-Resistant Staphylococcus aureus among Health Care Workers in Tertiary and Regional Hospitals in Dar es Salam, Tanzania. International Journal of Microbiology, 2018, Article ID: 5058390. https://doi.org/10.1155/2018/5058390

[36] Legese, H., Kahsay, A.G., Kahsay, A., Araya, T., Adhanom, G., Muthupandian, S., et al. (2018) Nasal Carriage, Risk Factors and Antimicrobial Susceptibility Pattern of Methicillin Resistant Staphylococcus aureus among Healthcare Workers in Adigrat and Wukro Hospitals, Tigray, Northern Ethiopia. BMC Research Notes, 11, Article No. 250. https://doi.org/10.1186/s13104-018-3353-2

[37] Abimana, J.B., Kato, C.D. and Bazira, J. (2019) Methicillin-Resistant Staphylococcus aureus Nasal Colonization among Healthcare Workers at Kampala International University Teaching Hospital, Southwestern Uganda. Canadian Journal of Infectious Diseases Medical Microbiology, 2019, Article ID: 4157869.

https://doi.org/10.1155/2019/4157869

[38] Dulon, M., Peters, C., Schablon, A. and Nienhaus, A. (2014) MRSA Carriage among Healthcare Workers in Non-outbreak Settings in Europe and the United States: A Systematic Review. BMC Infectious Diseases, 14, Article No. 363. https://doi.org/10.1186/1471-2334-14-363

[39] Albrich, W.C. and Harbarth, S. (2008) Health-Care Workers: Source, Vector, or Victim of MRSA? The Lancet Infectious Diseases, 8, 289-301. https://doi.org/10.1016/S1473-3099(08)70097-5

[40] Shittu, A.O., Kaba, M., Abdulgader, S.M., Ajao, Y.O., Abiola, M.O. and Olatimehin, A.O. (2018) Mupirocin-Resistant Staphylococcus aureus in Africa: A Systematic Review and Meta-Analysis. Antimicrobial Resistance \& Infection Control, 7, Article No. 101. https://doi.org/10.1186/s13756-018-0382-5 
[41] Seah, C., Alexander, D.C., Louie, L., Simor, A., Low, D.E., Longtin, J., et al. (2012) MupB, A New High-level Mupirocin Resistance Mechanism in Staphylococcus aureus. Antimicrobial Agents and Chemotherapy, 56, 1916-1920. https://doi.org/10.1128/AAC.05325-11

[42] Kateete, D.P., Asiimwe, B.B., Mayanja, R., Mujuni, B., Bwanga, F., Najjuka, C.F., et al. (2019) Nasopharyngeal Carriage, spa Types and Antibiotic Susceptibility Profiles of Staphylococcus aureus from Healthy Children Less than 5 Years in Eastern Uganda. BMC Infectious Diseases, 19, Article No. 1023. https://doi.org/10.1186/s12879-019-4652-5

[43] Iguchi. S., Mizutani, T., Hiramatsu, K. and Kikuchi, K. (2016) Rapid Acquisition of Linezolid Resistance in Methicillin-Resistant Staphylococcus aureus. Role of Hypermutation and Homologous Recombination. PLoS One, 11, e0155512.

https://doi.org/10.1371/journal.pone.0155512

[44] Mittal, G., Bhandari, V., Gaind, R., Rani, V., Chopra, S., Dawar, R., et al. (2019) Linezolid Resistant Coagulase Negative Staphylococci (LRCoNS) with Novel Mutations Causing Blood Stream Infections (BSI) in India. BMC Infectious Diseases, 19, Article No. 717. https://doi.org/10.1186/s12879-019-4368-6

[45] Barros, E.M., Martin, M.J., Selleck, E.M., Lebreton, F., Sampaio, J.L.M. and Gilmore, M.S. (2019) Daptomycin Resistance and Tolerance Due to Loss of Function in Staphylococcus aureus dsp1 and asp23. Antimicrobial Agents and Chemotherapy, 63, e01542-18. https://doi.org/10.1128/AAC.01542-18

[46] Rossi, C.C., Pereira, M.F. and Giambiagi-deMarval, M. (2020) Underrated Staphylococcus Species and their Role in Antimicrobial Resistance Spreading. Genetics Molecular Biology, 43, e20190065.

https://doi.org/10.1590/1678-4685-gmb-2019-0065

[47] Darboe, S., Dobreniecki, S., Jarju, S., Jallow, M., Mohammed, N.I., Wathuo, M., et al. (2019) Prevalence of Panton-Valentine Leukocidin (PVL) and Antimicrobial Resistance in Community-Acquired Clinical Staphylococcus aureus in an Urban Gambian Hospital: A 11-Year Period Retrospective Pilot Study. Frontiers in Cellular and Infection Microbiology, 9, 170. https://doi.org/10.3389/fcimb.2019.00170

[48] Young, B.C., Earle, S.G., Soeng, S., Sar, P., Kumar, V., Hor, S., et al. (2019) Panton-Valentine Leucocidin Is the Key Determinant of Staphylococcus aureus Pyomyositis in a Bacterial GWAS. Elife, 8, e42486.

https://doi.org/10.3389/fcimb.2019.00170

[49] Shallcross, L.J., Fragaszy, E., Johnson, A.M. and Hayward, A.C. (2013) The Role of the Panton-Valentine Leucocidin Toxin in Staphylococcal Disease: A Systematic Review and Meta-Analysis. Lancet Infectious Diseases, 13, 43-54. https://doi.org/10.1016/S1473-3099(12)70238-4

[50] Egyir, B., Guardabassi, L., Esson, J., Nielsen, S.S., Newman, M.J., Addo, K.K., et al. (2014) Insights into Nasal Carriage of Staphylococcus aureus in an Urban and a Rural Community in Ghana. PLoS ONE, 9, e96119.

https://doi.org/10.1371/journal.pone.0096119

[51] Moore, P. and Lindsay, J. (2002) Molecular Characterisation of the Dominant UK Methicillin-Resistant Staphylococcus aureus Strains, EMRSA-15 and EMRSA-16. Journal of Medical Microbiology, 51, 516-521. https://doi.org/10.1099/0022-1317-51-6-516

[52] Government of Kenya (2017) National Policy for the Prevention and Containment of Antimicrobial Resistance. Government of Kenya, Nairobi. 